\title{
Diseño, Desarrollo y Evaluación del MOOC "Introducción a la Gestión de Proyectos"
}

\author{
Alberto Palomares Chust ${ }^{a}$, Ignacio Despujol Zabala ${ }^{b}$ \\ ${ }^{a}$ Departamento de Sistemas Informático y Computación (DSIC), UPV, apalomares@dsic.upv.es \\ ${ }^{\mathrm{b}}$ Área de Sistemas de Información y Comunicaciones (ASIC), UPV, ndespujol@asic.upv.es
}

\section{Resumen}

En este artículo se analizan los aspectos más significativos del curso MOOC "Introducción a la Gestión de Proyectos" que la UPV imparte en EDX. La formación en Gestión de Proyectos es actualmente muy importante para todo tipo de empresas y organizaciones, y las perspectivas laborales para los profesionales formados en esta disciplina son muy buenas. Normalmente estos cursos suelen ser bastante caros e inaccesibles para muchos estudiantes, y por lo tanto es necesario desarrollarlos desde las Universidades Públicas (Servicio Público). Por otra parte, desde EDX tenían un interés estratégico preferente por el desarrollo de cursos en español en este tipo de disciplinas, para su expansión en toda el área de Sudamérica. Uno de los aspectos más innovadores de este curso MOOC es que se ha aplicado una metodología de mejora continua, es decir, se ha utilizado la información recogida en cada una de las ediciones para intentar mejorar la siguiente edición (corrección de errores, nuevos contenidos, etc.). Hasta el momento se han desarrollado 3 ediciones y la $4^{a}$ está prevista para empezar en Junio del 2017. Los resultados del curso son bastante buenos y además han ido mejorando en cada edición ( $n^{\circ}$ alumnos, certificados emitidos, ingresos, opiniones, etc.).

Palabras clave: MOOC, UPVX, EDX, Gestión de Proyectos, Project Management Institute (PMI) 


\section{Introducción}

Los MOOC (Massive Open Online Course) son cursos que se realizan en línea, dirigidos a un amplio número de participantes, en principio ilimitado, además abiertos a todo el mundo, y en muchos casos gratuitos. Los MOOC pueden ser clasificados en tres grupos principales: basados en la red de aprendizaje, basados en las tareas a desarrollar y basados en el contenido (Lane, 2012). Los MOOC basados en el contenido, conocidos como xMOOC, son los que más éxito han tenido (Daniel, 2012).

Los primeros cursos que pueden considerarse MOOC's propiamente dichos datan del año 2008. Desde entonces están suponiendo una verdadera revolución en la educación superior, y de manera complementaria, tambien en los planes de formación para empresas y organizaciones de todo tipo. Se estima que a finales del año 2016 unas 700 universidades de todo el mundo habían producido unos 7000 MOOC's en los que se habían matriculado unos 60 millones de estudiantes (Shah, 2016). El año 2012 fue denominado "año del MOOC" por diferentes medios de comunicación como el New York Times (Pappano, 2012).

La Universidad Politécnica de Valencia (UPV) estableció en el año 2007 un Plan de actuación llamado Plan de Docencia en Red $^{1}$, que pretendía favorecer el uso de las TIC en la práctica docente, apoyando al profesor en todo los procesos relacionados con acciones innovadoras y proporcionando tanto recursos como servicios de apoyo que facilitaran el uso de estas tecnologías en la práctica docente.

En este contexto se ha desarrollado el sistema Polimedia, que permite grabar vídeos docentes de alta definición de forma rápida y sencilla, usando unos estudios audiovisuales de bajo coste (Turró, Cañero \& Busquets, 2010). Estos proyectos han permitido ir consolidando a la UPV como una institución de referencia en la elaboración de materiales docentes on-line ${ }^{2}$, y toda esta experiencia acumulada ha sido fundamental para participar activamente en el desarrollo de MOOC's (Despujol, 2014).

Los cursos MOOC requieren de Plataformas Tecnológicas desarrolladas específicamente para poder satisfacer las necesidades de este tipo de cursos: proporcionar a los alumnos los materiales necesarios para el curso (documentos, exámenes, videos, etc.), foros que les permiten interaccionar entre sí y con el profesorado que los imparte, etc. Algunas de las plataformas más conocidas de MOOC's son EDX, Udacity, Open2study, Coursera, etc.

La plataforma EDX $^{3}$, desarrollada por la Universidad de Harvard y el Instituto Tecnológico de Massachusetts (MIT), se ha consolidado, desde su aparición el año 2012, como uno de los principales proveedores de cursos MOOC de alta calidad en el mundo.

\footnotetext{
1 https://www.upv.es/contenidos/DOCENRED/indexc.html

2 DATOS GENERALES (Marzo 2017): unos 16.875 objetos de aprendizaje grabados, más de 1.000 profesores involucrados y un canal educativo en Youtube (6.380 vídeos, 87.000 suscriptores y 22,8 millones de visualizaciones)

3 https://www.edx.org/
} 
Algunas de las principales instituciones universitarias del mundo están adheridas e imparten sus cursos MOOC en esta plataforma: Harvard, MIT, Berkeley, Sorbonne, Imperial College London, ETH Zurich, Technische Universitat Munich, Universiyty Chicago, University of Edinburgh, etc. EDX ha superado los 10 millones de usuarios únicos (datos de febrero $2017)^{4}$.

Una característica de los cursos que se ofertan en EDX es que los alumnos de todo el mundo pueden acceder libre y gratuitamente a todos los contenidos, y por lo tanto también permiten a empresas y organizaciones diseñar programas de formación para sus empleados. Los alumnos que realizan un curso completo, realizan los exámenes y obtienen la calificación mínima exigida para aprobar el curso, pueden optar a obtener un certificado que expide EDX, que tiene un determinado coste (por ejemplo para el MOOC que se describe en este artículo es de 50 \$). Es decir, la formación propiamente dicha es gratuita y solamente la certificación tiene un pequeño coste para los alumnos. Las denominadas Inscripciones Verificadas son las que optan por un certificado verificado y pagan por su expedición.

La UPV está adherida a EDX desde Noviembre del 2014, siendo una de las primeras universidades españolas en impartir sus cursos MOOC en esta prestigiosa plataforma. La UPV dispone de una versión local de plataforma denominada UPVX (http://upvx.es/). Uno de los objetivos de UPVX es poder lanzar las primeras versiones de los cursos MOOC para poder testearlos, ver el grado de aceptación, corregir errores, etc. Es decir, UPVX permite hacer un control de calidad de estos cursos, ya que la Plataforma EDX es muy exigente en cuanto a los requisitos de calidad que deben cumplir los cursos para ofertarlos. Durante el año 2015 ya se impartieron más de 30 cursos MOOC de la UPV en EDX, siendo la institución con mayor crecimiento en cursos MOOC en la plataforma. Además es una de las exitosas por lo que se refiere a la tasa de finalización de los mismos, un 9,6\%, muy por encima del aproximadamente 5\% de la media de los cursos en EDX.

Entre los MOOC que la UPV imparte en EDX está el curso "Introducción a la Gestión de Proyectos", que ha sido desarrollado por Alberto Palomares Chust, Profesor del Departamento de Sistemas Informáticos y Computación (DSIC) de la UPV, adscrito a la Escuela Politécnica Superior de Gandía (EPSG), y además es miembro del Project Management Institute (PMI) ${ }^{6}$, socio del Capítulo de Valencia del $\mathrm{PMI}^{7}$ y con la certificación Project Management Professional (PMP) ${ }^{8}$.

\footnotetext{
4 http://blog.edx.org/celebrating-10-million-edx-learners-worldwide

5 https://courses.edx.org/courses/course-v1:UPValenciax+IGP101.x+2T2016/info

${ }^{6}$ El PMI es una Organización sin ánimo de lucro que promueve la profesión de la Dirección de Proyectos mediante la definición y publicación de estándares, la promoción de certificaciones profesionales mundialmente reconocidas, la dinamización de comunidades colaborativas, la financiación de programas de investigación y la potenciación de oportunidades de desarrollo profesional. Actualmente es la principal organización a nivel internacional en el campo de la Gestión de Proyectos, y tiene unos 500.000 socios de prácticamente todos los países del mundo http://www.pmi.org/.

7 El PMI está presente en casi todo el mundo a través de sus Capítulos nacionales o regionales http://www.pmi-valencia.org/

${ }^{8}$ La certificación PMP es la más reconocida a nivel internacional en el campo de la Gestión de Proyectos.
} 
El motivo principal para empezar a desarrollar este MOOC fue el hecho de que la formación en Gestión de Proyectos es actualmente de una gran importancia para todo tipo de empresas y organizaciones, de muchos sectores, y a nivel internacional. Las perspectivas para los profesionales formados en Gestión de Proyectos son muy buenas, tanto por las mejores posibilidades para encontrar empleos como por la calidad/salarios de dichos empleos.

Según el informe que elabora anualmente el PMI "PMI's Pulse of the Profession" 9, "la gestión de proyectos es esencial para el éxito de las organizaciones. Está demostrado que aquellas organizaciones que invierten en Gestión de Proyectos obtienen unos resultados mejores que aquellas que no lo hacen, ya que consiguen finalizar más proyectos cumpliendo plazos, costes, criterios de calidad, etc". Empresas y organizaciones de casi cualquier sector y prácticamente en todos los países del mundo requieren profesionales formados en Gestión de Proyectos.

Es importante poner de manifiesto que en general es difícil encontrar cursos en el campo de la Gestión de Proyectos asequibles para una parte significativa de la población, ya que los cursos suelen ser bastante caros. Esta es una razón fundamental para desarrollar este tipo de formación desde una Universidad Pública como la UPV.

\section{Objetivos}

El objetivo de este curso (tal y como aparece textualmente en la descripción en EDX) es el siguiente: "Introducir algunos conceptos básicos de la Gestión de Proyectos / Project Management (PM), según las metodologías del Project Management Institute (PMI). El PMI es la Principal Organización Mundial en el campo de la Dirección de Proyectos (www.pmi.org). Sus guias están basadas en las "Buenas Prácticas" de profesionales de todo el mundo, y sus certificaciones, por ejemplo el Project Manager Professional (PMP) son muy demandadas por empresas de todos los sectores".

Este MOOC es un curso muy básico de Gestión de Proyectos (GP) y no se requieren conocimientos específicos previos para poder realizarlo. Consta de 4 Unidades.

1. Introducción a la Gestión de Proyectos

2. Gestión del Tiempo de un Proyecto

3. Gestión de Riesgos de un Proyecto

4. $\quad$ Final (exámenes)

\footnotetext{
${ }^{9}$ http://www.pmi.org///media/pmi/documents/public/pdf/learning/thought-leadership/pulse/pulse-of-the-profession-2017.pdf
} 


\section{Desarrollo de la innovación}

La preparación de este curso estaba todavía en una fase intermedia cuando los responsables de EDX comunicaron a la UPV su interés preferente por el desarrollo de cursos en español relacionados con ciertos campos en particular, por ejemplo Emprendimiento, Negocios, Gestión de Proyectos, etc. El motivo concreto era su importancia estratégica para la expansión de EDX en toda el área de Sudamérica.

Esta es una de las razones que impulsaron y aceleraron el desarrollo de este curso, adelantando los plazos previstos inicialmente para su producción y publicación. Hasta el momento se han desarrollado las siguientes ediciones:

- La primera edición del MOOC se desarrolló en la plataforma UPVX. Periodo programado: Junio-Julio 2015.

- La segunda edición del MOOC se desarrolló en la plataforma EDX. Periodo programado: Noviembre-Diciembre 2015.

- La tercera edición del MOOC se desarrolló en la plataforma EDX. Periodo Programado: Junio-Julio 2016.

- Tercera edición (Self Paced) ${ }^{10}$ la tercera edición ha seguido estando disponible en EDX hasta su cierre definitivo en Junió de 2017.

- La cuarta edición está programada para empezar en Junio del 2017.

Uno de los aspectos más innovadores en el desarrollo de este curso MOOC es que se ha aplicado una metodología de mejora continua, es decir, se ha recogido y analizado la información generada por los alumnos en los foros de la plataforma en cada una de las ediciones (opiniones, preguntas, dudas, problemas, etc) y esta información se ha utilizado para corregir algunos errores, incorporar algunos contenidos adicionales, crear debates específicos en los foros sobre algunos temas en los que los alumnos suelen tener más problemas, etc. Por lo tanto el curso MOOC ha ido mejorando sus contenidos en cada una de las ediciones.

\footnotetext{
${ }^{10}$ Es lo que se denomina en modo "Self Paced" que se puede traducir como "a su ritmo", para que los alumnos puedan realizarlo por su cuenta, pero sin el soporte contínuo de los profesores en los foros que se realiza en los periodos programados.
} 


\section{Resultados}

En el próximo apartado se presentan diferentes datos para mostrar el grado de difusión y aceptación del MOOC, en sus diferentes ediciones, especialmente de la tercera que es la más reciente en el momento de escribir este artículo.

\section{$3^{a}$ Edición (Junio-Julio 2016). EDX. Resultados Cuantitativos.}

Inscripciones / Inscripciones Verificadas

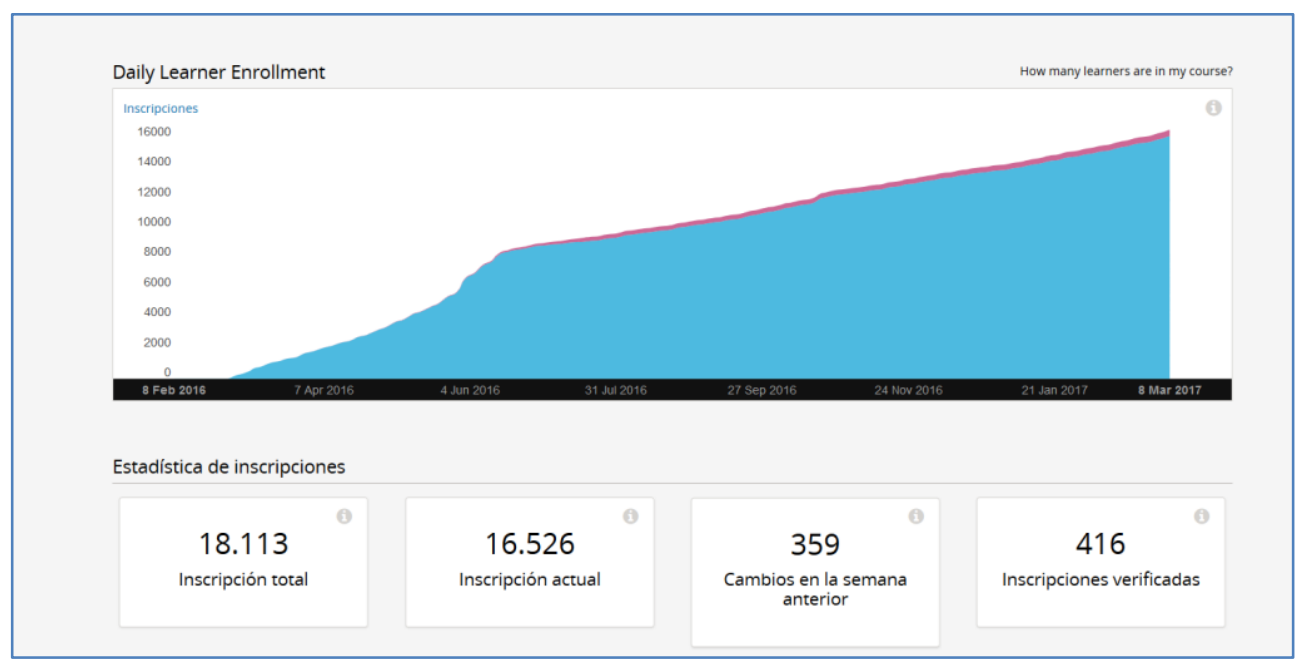

Figura 1: inscripciones/inscripciones verificadas de los alumnos

Edad de los alumnos



Figura 2: edad de los alumnos 


\section{Nivel de Estudios}

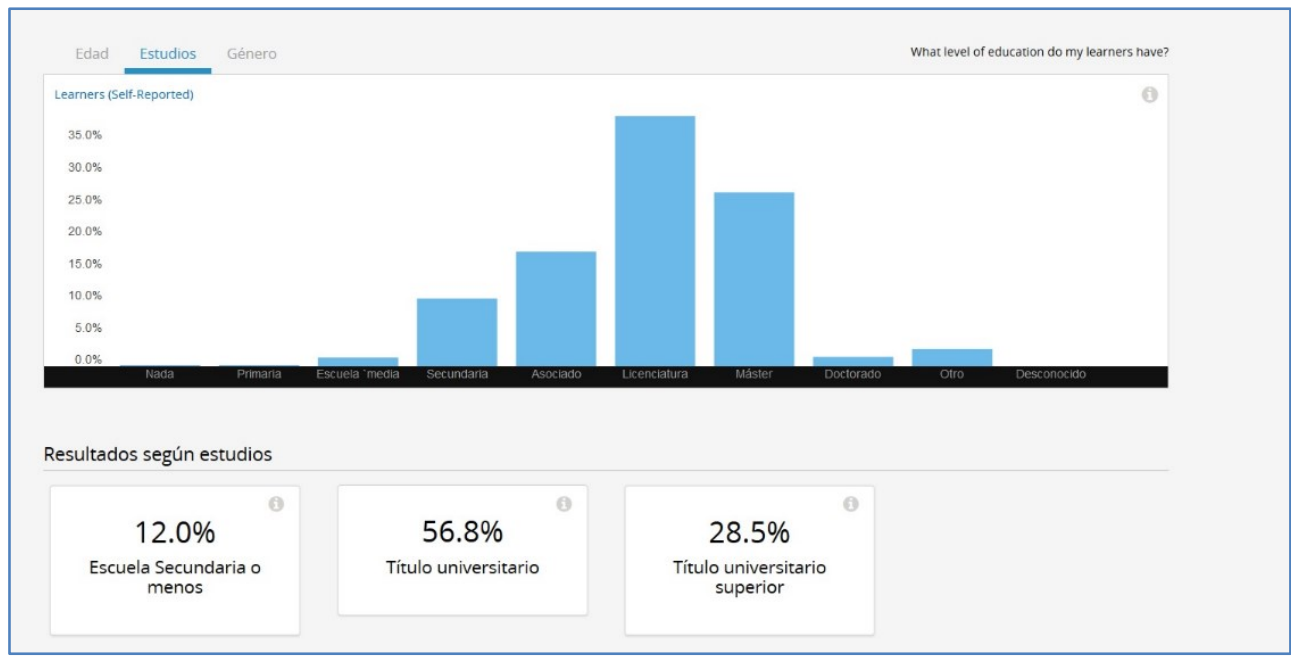

Figura 3: nivel de estudios de los alumnos

Distribución Geográfica de los Alumnos



Figura 4: distribución geográfica de los alumnos

\begin{tabular}{|l|l|}
\hline$(c)$ EY-NC-ND 2017, UPV \\
\hline
\end{tabular}

Congreso In-Red (2017) 


\section{$3^{a}$ Edición (Junio-Julio 2016). EDX. Resultados Encuestas ${ }^{11}$}

Sugerencias de los Alumnos (texto libre)



Figura 5: Sugerencias de los alumnos (nube de palabras realizada a partir de 470 respuestas)

Opinión sobre la Frecuencia de Publicación y Ritmo de Aprendizaje del Curso

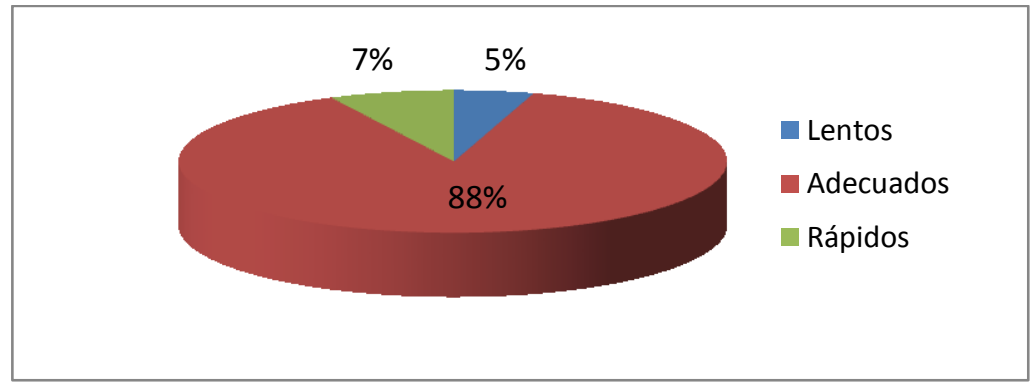

Figura 6: Frecuencia de Publicación y Ritmo de Aprendizaje (calculada a partir de 1091 respuestas)

Opinión sobre la Duración de los Videos

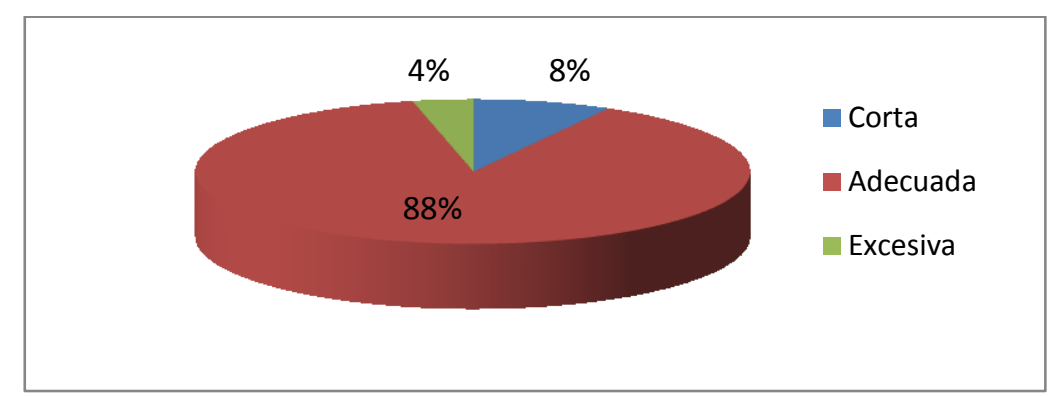

Figura 7: Duración de los Videos (calculada a partir de 1080 respuestas)

\footnotetext{
${ }^{11}$ Respuestas proporcionadas por los alumnos en las encuestas realizadas al finalizar el curso
} 
Opinión sobre el Contenido de los Videos

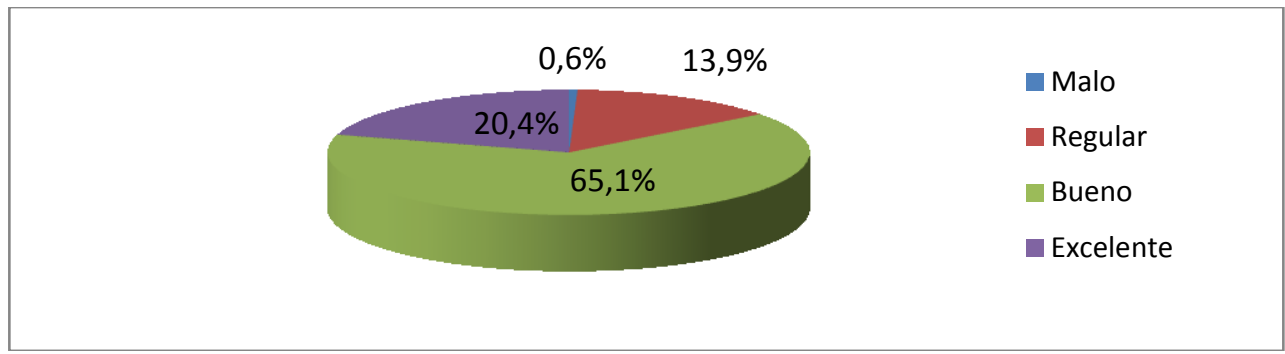

Figura 8: Contenido de los Videos (calculada a partir de 1069 respuestas)

Opinión sobre Exámenes y Actividades

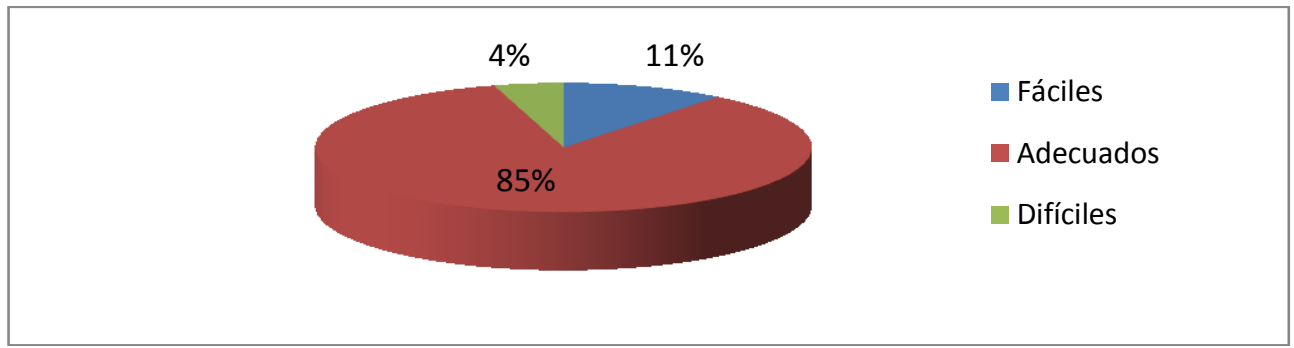

Figura 9: Exámenes y Actividades (1065 respuestas)

Opinión sobre el Nivel del Curso



Figura 10: Nivel del Curso (1093 respuestas)

Opinión sobre la Tutorización (Resolución de Dudas)

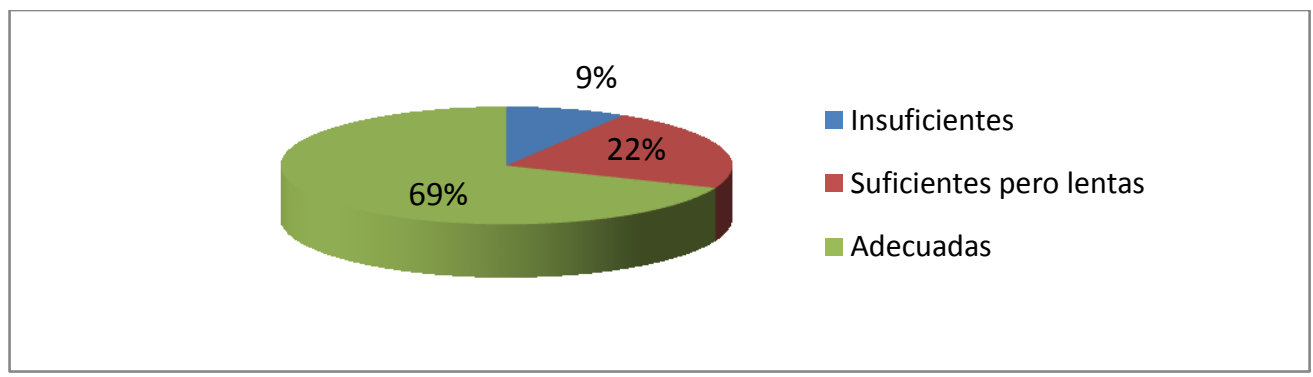

Figura 11: Tutorización (1054 respuestas) 
Opinión sobre el Aprendizaje

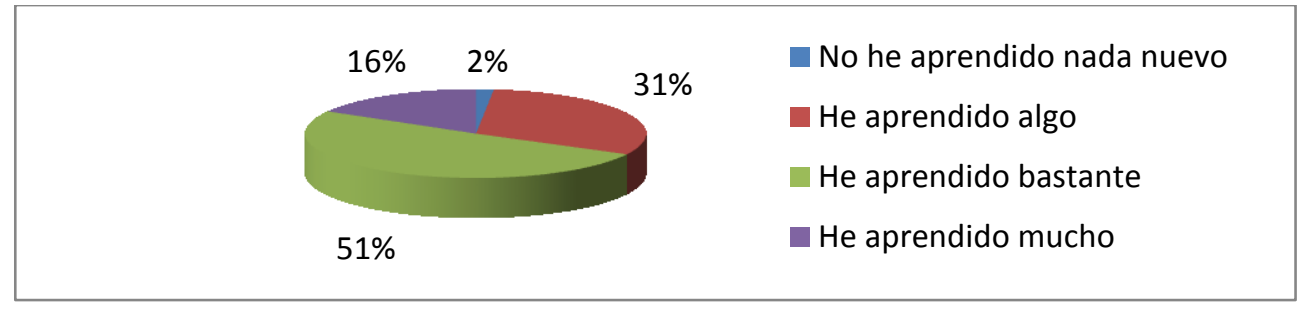

Figura 12: Aprendizaje (1076 respuestas)

Opinión sobre el Sistema de Aprendizaje



Figura 13: Sistema de Aprendizaje (1088 respuestas)

Opinión sobre Expectativas del Curso (valoración del 1 al 5)

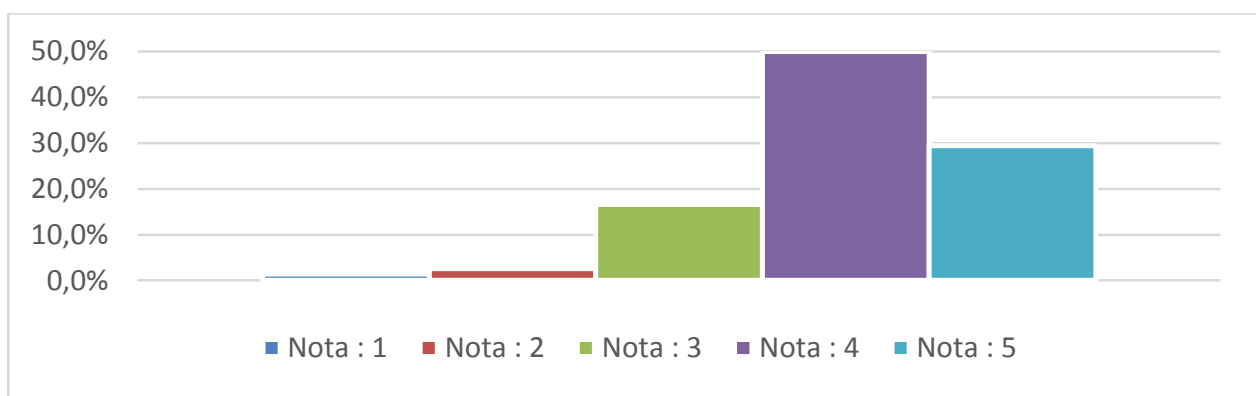

Figura 14: Expectativas (1035 respuestas)

Opinión sobre Dedicación (¿Cuántas horas le han dedicdo al curso?)

Este punto es muy importante ya que los alumnos quieren saber "a priori" el tiempo que le tienen que dedicar al curso. Los resultados son los siguientes (1027 respuestas):

- $\quad$ Entre 1 y 3 horas: $49,03 \%$

- $\quad$ Entre 3 y 5 horas: $22,86 \%$

- $\quad$ Entre 5 y 7 horas: 9,68\%

Estos datos nos indican que una recomendación bastante adecuada, para la mayoría de los alumnos que realizan el curso, es que con una dedicación de unas 4-5 horas semanales durante las 5-6 semanas planificadas para realizar el curso es suficiente para poder aprobarlo. Esta información se utiliza en las recomendaciones que se hacen a los nuevos 
alumnos sobreel tiempo que tienen que dedicar para realizar el curso (información basada en los datos).

\section{$2^{a}$ Edición (Noviembre-Diciembre 2015). EDX}

Inscripciones / Inscripciones Verificadas

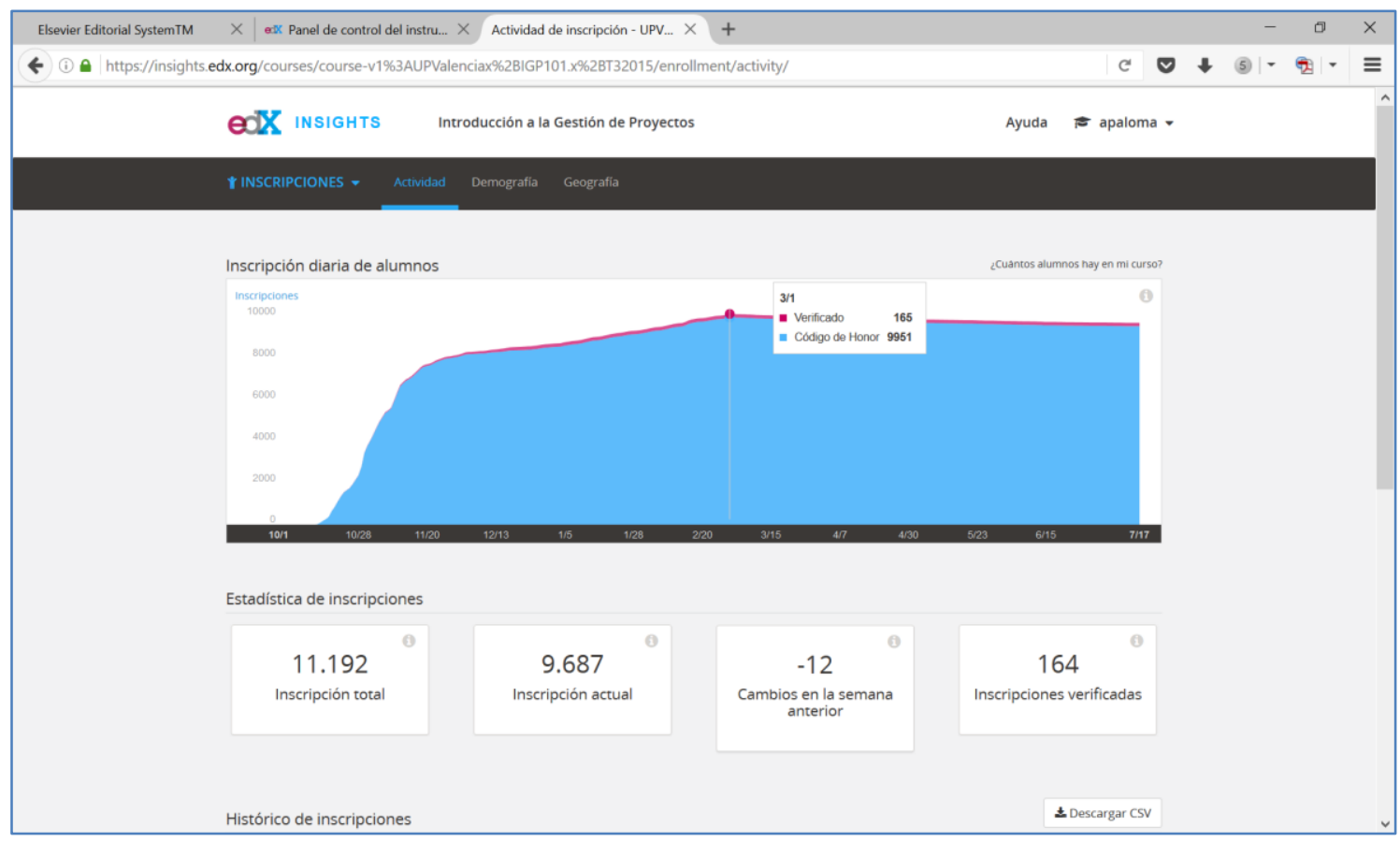

Figura 15: inscripciones/inscripciones verificadas de los alumnos

Distribución Geográfica / Edad / Nivel de Estudios de los Alumnos

Los datos correspondientes a la $2^{\text {a }}$ edición son muy similares a los de la tercera edición, que se describen en el apartado anterior, y por esta razón no se representan.

\section{$1^{a}$ Edición (Junio-Julio 2015). UPVX}

Esta edición se relizo en UPVX y sólo se muestran algunos datos básicos del curso:

- $\quad \mathrm{N}^{\mathrm{o}}$ de Alumnos Matriculados: 3193

- $\quad \mathrm{N}^{0}$ de Alumnos que han Interactuado con el Curso: 992

- $\quad \mathrm{N}^{0}$ de Alumnos Aprobado el Curso: 542 (Certificado UPV Gratuito). 


\section{Conclusiones}

La conclusión principal es que este curso, en general, está teniendo unos resultados bastante buenos (con respecto a otros cursos MOOC) y además, y quizás lo que es más importante, es que estos resultados han ido mejorando en cada una de las ediciones del curso. Consideramos que la mejora de los resultados se debe principalmente a que se ha aplicado un proceso de mejora continua. A continuación se describen brevemente algunos de los principales resultados del curso:

El número de alumnos inscritos en el curso (en las 3 ediciones) está por encima de los 30.000 , de los cuales aproximadamente unos 600 han realizado la inscripción verificada en EDX, es decir, han pagado los $50 \$$ requeridos para obtener el certificado del curso. Por lo tanto, los ingresos totales del curso en la plataforma están actualmente por encima de los $30.000 \$$.

El perfil de un alumno del curso es hispanoamericano ${ }^{12}$, titulado superior, con una edad por encima de los 25 años. Es decir, básicamente es un alumno posgraduado, probablemente trabajando o quizás buscando su primer empleo, pero que necesita formación adicional en el campo de la Gestión de Proyectos para mejorar su empleo actual o para encontrarlo.

Este perfil de los estudiantes del curso, coincide, en líneas generales, con el que la literatura describe para otros cursos MOOC. Así, por ejemplo, un estudio sobre los estudiantes inscritos en los cursos que la Universidad de Pennsylvania desarrolla en la plataforma Coursera confirma el predominio de alumnos con estudios superiores Emanuel (2013). Es decir, los MOOCs no están llegando a estudiantes muy desfavorecidos económicamente y con muy pocas oportunidades educativas como quizás se podría suponer a priori. Para realizar estos cursos los alumnos tienen que tener un nivel digital aceptable Yuan\&Powell (2013).

Tal y como se puede comprobar en los propios foros que la plataforma EDX proporciona, los alumnos consideran, de manera bastante generalizada, que este MOOC un buen curso, y los comentarios mayoritariamente son favorables. Ha habido alumnos que explícitamente han comentado que el curso les ha permitido mejorar profesionalmente, por ejemplo

http://blog.edx.org/quieres-mejorar-en-tu-carrera-profesional-y-no-sabes-como-2?track=blog

Desde EDX han informado a la UPV que este curso ( $3^{\text {a }}$ edición) es uno de los 10 que más éxito está teniendo a nivel mundial en el modo de funcionamiento "self-paced". En este sentido se debe destacar la participación del personal de apoyo de la UPV, los denominados "Servipolis", que permiten dinamizar los foros de la plataforma, facilitando el trabajo de los profesores, especialmente en la resolución de dudas genéricas (relacionadas con diferentes aspectos del uso de la plataforma EDX), pero también de dudas de contenido, ya que con el desarrollo de diferentes ediciones y su presencia en los foros, también están dominando la materia del curso.

${ }^{12}$ Los alumnos de Perú, Colombia, España, Mexico y Ecuador representan aproximadamente el $70 \%$ de los inscritos en el MOOC. 


\section{Referencias}

DANIEL, J. (2012). Making sense of MOOCs: Musings in a maze of myth, paradox and possibility. Journal of Interactive Media in Education. < $\underline{\text { http://www- }}$ jime.open.ac.uk/jime/article/view/2012-18>. [Consulta: 22 abril 2015].

DESPUJOL, I. (2014). Servicios TIC de apoyo a la docencia. Universitat Politècnica de València. < http://es.slideshare.net/ndespujol/presentacin-global-serviciosmmedia-asic-2014>. [Consulta: 21 julio 2015].

EMANUEL, E.J. (2013). Online education: MOOCs taken by educated few. Nature, 503(342). < http://dx.doi.org/10.1038/503342a >. [Consulta: 22 abril 2015].

LANE, L. (2012). Three kinds of MOOC. < http://lisahistory.net/wordpress/2012/08/threekinds-of-moocs/>. [Consulta: 22 abril 2015].

PAPPANO, L.. The Year of the MOOC. The New York Times. http://www.nytimes.com/2012/11/04/education/edlife/massive-open-onlinecourses-are-multiplying-at-a-rapid-pace.html? r=0. [Consulta: 2012, November].

SHAH, D. Monetization over Massiveness: A Review of MOOC Stats and Trends in 2016. Class Central. <https://www.class-central.com/report/moocs-stats-and-trends2016/>. [Consulta: 2016, December].

TURRO, C. CAÑERO A., \& BUSQUETS, J. (2010). Video learning objects creation with Polimedia. Proceedings of the 2010 IEEE International Symposium on Multimedia. isbn=978-0-7695-4217-1.

YUAN, L. \& POWEL, S. (2013). MOOCs and open education: Implications for higher education (JISC CETIS white paper). <http://publications.cetis.ac.uk/2013/667>. [Consulta: 22 abril 2015]. 